\title{
Comparison Aerodynamic Performance and Power Fluctuation Between Darrieus Straight-Bladed and Gorlov Vertical Axis Wind Turbines
}

\author{
M. Moghimi ${ }^{\dagger}$ and H. Motawej \\ School of Mechanical Engineering, Iran University of Science \& Technology, Tehran, Iran \\ †Corresponding Author Email: moghimi@iust.ac.ir
}

(Received August 5, 2019; accepted February 29, 2020)

\begin{abstract}
All human actions necessitate energy resources. Currently, a major part of our energy requirements is supplied by fossil fuels, which are faced with uncertainties concerning their availability in the forthcoming decades. However, the combustion of fuels results in adverse environmental aftermaths. Energy of wind falls into one of the clean and renewable energy resources. Wind power is generated using horizontal and vertical axis wind turbines (HAWTs \& VAWTs). VAWTs operate appropriately under low wind velocity conditions to generate power in small scales. On the other hand, numerous VAWT designs have been presented to enhance their performance in such circumstances. This study is aimed at developing cost-effective aerodynamic calculations models for both Gorlov and Darrieus straight-bladed VAWT types. Thus, DMST (double multiple stream tube) models, which act on the basis of BEM (blade element momentum) theory, have been designed for Gorlov and Darrieus VAWTs. By comparison of the results obtained with those available in the literature, the models developed are validated. Additionally, the performance of the Darrieus-type straight-bladed VAWTs was compared to that of the Gorlov VAWTs. According to the findings, although the peak power coefficient $\left(C_{P}\right)$ decreased slightly for Gorlov VAWTs in comparison with the Darrieus straight-bladed type, the Gorlov rotor showed improved performance in terms of fluctuation and effectiveness based on the torque coefficient curve of helical blades.
\end{abstract}

Keywords: Darrieus; VAWT; DMST; Gorlov; power fluctuation; Aerodynamic performance.

\section{NOMENCLATURE}

$\begin{array}{ll}A & \text { swept area } \\ \alpha & \text { local angle of attack } \\ a d & \text { downstream induction factor } \\ a u & \text { upstream induction factor } \\ a v & \text { average } \\ c & \text { blade chord } \\ C_{D} & \text { drag coefficient } \\ C_{L} & \text { lift coefficient } \\ C_{N} & \text { normal force coefficient } \\ C_{P} & \text { power coefficient } \\ C_{T} & \text { tangential force coefficient } \\ C_{q} & \text { torque coefficient } \\ d w & \text { downstream } \\ F_{N} & \text { normal force component } \\ F_{T} & \text { tangential force component } \\ H & \text { blade height } \\ H / 2 R & \text { aspect ratio } \\ N_{h} & \text { horizontal stream tubes number } \\ N_{v} & \text { vertical stream tubes number } \\ N & \text { Blades number } \\ Q & \text { instantaneous torque }\end{array}$

swept area

$\begin{array}{ll}R & \text { rotor radius } \\ R e & \text { local blade Reynolds number } \\ u p & \text { upstream } \\ V_{\infty} & \text { free wind velocity } \\ V_{a d} & \text { downstream induced velocity } \\ V_{a u} & \text { upstream induced velocity } \\ V_{e} & \text { equilibrium induced velocity } \\ W & \text { local relative velocity } \\ & \\ \lambda_{0} & \text { local tip speed ratio } \\ \Delta h & \text { stream tube height } \\ \Delta \theta & \text { blade azimuth angle variation } \\ \Psi & \text { helical angle } \\ \theta & \text { azimuth angle } \\ \lambda & \text { turbine tip speed ratio } \\ v & \text { kinematic viscosity of air } \\ \rho & \text { air density } \\ \sigma & \text { solidity } \\ \omega & \text { Rotational speed }\end{array}$




\section{INTRODUCTION}

Despite the fact that fossil fuels account for the major source of energy all over the world today, these sources are faced with great uncertainties in the next decades. In truth, due to their greater consumption rates, the planet may run out of these fuels in the forthcoming years. Furthermore, the fossil fuels consumption is of increasing adverse impacts on environment. For example, since the uncovering of the fact that the greenhouse gases are among the key contributors to global warming, they have been regarded as a serious issue for humans (Moghimi et al. 2018). On September 25, 2015, the United Nations (UN) disseminated a promising proposal stating that an expectable ideal universe will be realized by 2030. Such an aspiring ambition is dependent upon 17 sustainable development objectives, among which the $7^{\text {th }}$ goal focuses on guaranteeing global accessibility of unpolluted, inexpensive, and dependable energy, and the $13^{\text {th }}$ goal focuses on combating climate change (UN News Centre, 2015). Thus, there is a need for finding free, widely available, and clean energy resources that replace the fossil fuels in the years to come. Wind energy accounts for one of the renewable sources of energy, and thus, a variety of wind turbines have been developed to generate electrical energy (Zhu and Liu, 2018). Recently, the growing demand for energy has accelerated the design of wind turbines (Noura et al. 2016). Depending on the axis direction of rotor, one can categorize wind turbines into two major groups: VAWTs (vertical axis wind turbines) and HAWTs (horizontal axis wind turbines). Today, the unquestionable market leader is the HAWT technology, which has been utilized even in offshore sites in recent years (Derakhshan et al. 2018). Based on their low wind speed demands, VAWTs are generally utilized not only in distant sites, but in urban and rural environments as well (Dilimulati et al. 2018). Some of the advantages offered by the VAWT technology are their simple structure, independency of wind direction due to their being a cross-flow device to receive wind from all directions, their no need for yaw mechanism, withstanding highly turbulent winds, less noise pollution because of lower tip speed ratios, and more convenient maintenance, because one may place the generator on the ground (Sagharichi et al. 2018). The VAWT concept has evolved over time into a number of mature designs, which are utilized currently. An anemometer has been the source of inspiration for Savonius VAWT, and it utilizes "S"shaped scoops, thereby generating sufficient drag for rotation, whereas the Darrieus VAWT, given its lift-based configuration, is of greater coefficients of power and more commonly application (AbuHamdeh and Almitani, 2017). Darrieus VAWTs suffer from some drawbacks including lower efficiency, periodic variations of aerodynamic forces throughout each rotation cycle, and poor selfstarting (Zamani et al. 2016). Professor A.M. Gorlov from Northeastern University with expertise in hydropower designed the Gorlov turbine, which received US Patents no. 5,451,137 of 1995 (Gorlov, 1995). To decrease the periodically unsteady torques faced in straight-bladed concepts, the cross-flow axis configuration has been designed with helical blades. Despite the fact that the turbine was primarily of a water turbine design, the released patents declared that it was usable for hydro, hydro-pneumatic, wave and wind power systems. A number of authors have analyzed Gorlov VAWTs through CFD (computational fluid dynamics) simulation. Through three dimensional U-RANS (unsteady Reynoldsaveraged Navier-Stokes) simulation, Alaimo et al. (2015) made a comparison between helical- and straight-bladed VAWTs in terms of their aerodynamic performance. They found that although power generation by a helical rotor was lower, its performance was more stable compared to that of a straight-bladed one. In order to predict the output power of a helical VAWT in various tip speed ratios, Cheng et al. (2017) applied two dimensional LES (large-eddy simulation). The authors suggested for future studies to decrease or eliminate three dimensional impacts on aerodynamic performance of turbines. According to few existing literatures, the perfect performance of Gorlov turbines is attributable to their curved blades with helical arrangement around the axis of rotor. Thus far, however, one can find scarce performance data on Gorlov turbines in previous research, particularly with regard to wind energy. The whole above findings reflect the fact that detailed analysis of performance of Gorlov VAWT requires more insight. The VAWTs performance has been estimated using various computational models. Studies conducted experimentally (Amiri et al. 2019; Battisti et al. 2018; Peng et al. 2019) and numerically according to CFD techniques (Balduzzi et al. 2016; Rogowski et al. 2018; Zhu et al. 2019) investigated the VAWTs aerodynamic performance that were applied successfully to characterize the VAWTs. It is crucial to accurately predict the output power of wind turbines to estimate the generator size and also other mechanical elements; thus, the BEM (blade element momentum) theory has been introduced. Momentum models (e.g. stream tube models) offer a popular and efficient technique for the performance prediction and designing of the wind turbines. Moreover, an acceptable consistency was reported between the results of these models and those obtained experimentally (Bedon et al. 2014). Also, a good agreement between CFD results and those of above models was reported by Balduzzi et al. (2017). Given the appropriate behavior of DMST (double multiple stream tube) model in aerodynamic calculations of VAWT as well as the fast prediction, the model has been widely applied in earlier researches to analyze the VAWTs performance, and some studies have confirmed its reliability to capture the behavior of VAWTs (Abdul Akbar and Mustafa, 2016; Jafari et al. 2018; Meana-Fernández et al. 2018; Moghimi and Motawej, 2020).

The current research develops and uses the DMST models for the comparison and evaluation of the power and torque coefficients of both Gorlov and Darrieus straight-bladed VAWT types. 


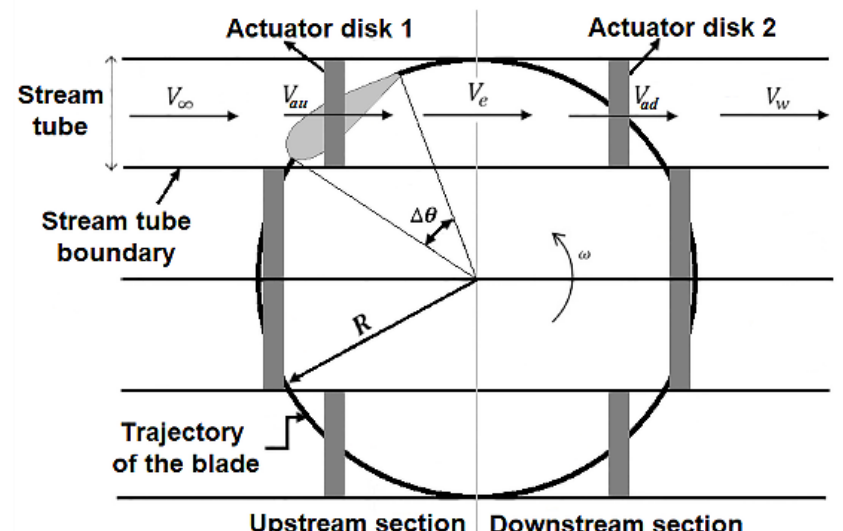

Upstream section Downstream section

Fig. 1. Sketch of upstream and downstream section in DMST model.

\section{MATERIALS AND METHODS}

\subsection{DMST (Double Multiple Stream Tube) Model}

In the BEM (blade element momentum) model, the momentum theory is merged with the blade element theory, and one can use the model to analyze the forces acting on blades and flow behavior (Paraschivoiu, 2002). In the same model, a theoretical disk is used to model the turbine extracting the wind energy because of the drag force imposed by the inflow. First, it is assumed that the wind is decelerated by the disk/turbine. Then, the drag imposed by the wind on the turbine is estimated and a comparison is made with the disk drag via the blade element approach for the assumed velocity. Finally, it is possible to calculate the aerodynamic variables, the turbine performance and the wind velocity profiles upon meeting the convergence criterion (Meana-Fernández et al. 2018). According to the BEM theory, multiple models have been designed. Templin (1974) was the first to propose the SST (single stream tube) model in which the turbine is mounted within a unique stream tube surrounding the entire turbine. Designed by Strickland (1975), the MST (multiple stream tube) model is consisted of multiple stream tubes placed in parallel next to each other. Paraschivoiu (1981) introduced the DMST (double multiple stream tube) model, which tries to eliminate the incapability of the MST model to distinguish between the upstream and downstream sections of a rotor. Thus, the actuator disc of the turbine is partitioned to two parts that represent the upwind section $(-\pi / 2<\theta<\pi / 2)$ and the downwind section $(\pi / 2<\theta<3 \pi / 2)$ as shown in Fig. 1. This method can model the flow variations within the wind turbine more accurately.

The stream tubes direction in DMST model is axial, therefore the free stream velocity, $V_{\infty}$, is decreased by induction factors of upstream $a u$ and downstream $a d . V_{a u}$ is the upstream induced velocity and $V_{a d}$ is the downstream induced velocity. While, $V_{e}$ represents the equilibrium induced velocity. The $V_{a u}$,
$V_{e}$, and $V_{a d}$ are obtained by (Jafari et al. 2018; Paraschivoiu, 1981; Tahani et al. 2016):

$$
\begin{aligned}
& V_{a u}=a u V_{\infty} \\
& V_{e}=(2 a u-1) V_{\infty} \\
& V_{a d}=a d(2 a u-1) V_{\infty}
\end{aligned}
$$

One can calculated the tangential and normal coefficients, $C_{T}$ and $C_{N}$ using the equations below (Zhao et al. 2017):

$$
\begin{aligned}
& C_{N}=C_{L} \cos \left(\alpha_{u p}\right)+C_{D} \sin \left(\alpha_{u p}\right) \\
& C_{T}=C_{L} \sin \left(\alpha_{u p}\right)-C_{D} \cos \left(\alpha_{u p}\right)
\end{aligned}
$$

where $C_{L}$ and $C_{D}$ are the lift and drag coefficients, and the local angle of attack is represented by $\alpha_{u p}$.

For every stream tube, by using BEM theory, the induction factor is estimated for the upstream section, au, (Kavade and Ghanegaonkar, 2018; Saeidi et al. 2013):

$$
a u=\frac{\pi}{F_{u p}+\pi}
$$

where $F_{u p}$ denotes the upwind circumstances defined as:

$$
\begin{aligned}
& F_{u p} \\
& =\frac{N c}{8 \pi R} \int_{-\pi / 2}^{\pi / 2}\left(\frac{W_{u p}}{V_{a u}}\right)^{2}|\sec (\theta)|\left(C_{N} \cos (\theta)\right. \\
& \left.-C_{T} \sin (\theta)\right) d \theta
\end{aligned}
$$

where $c$ is the blade chord, $\theta$ represents the azimuth angle, $N$ shows the number of blades, $W_{u p}$ is the local relative velocity, and $R$ represents the rotor radius.

The tangent $F_{T}$ and normal $F_{N}$ are components of the resultant force of the rotor (Fig. 2). The $F_{T}$ and $F_{N}$ are determined as functions of the azimuth angle $\theta$ by (Batista et al. 2018; Hashem and Mohamed, 2018):

$$
F_{N}(\theta)=\frac{1}{2} C_{N} \rho c \Delta h W_{u p}^{2}
$$




$$
F_{T}(\theta)=\frac{1}{2} C_{T} \rho c \Delta h W_{u p}^{2}
$$

in which, $\Delta h$ is the stream tube height and $\rho$ is the air density.

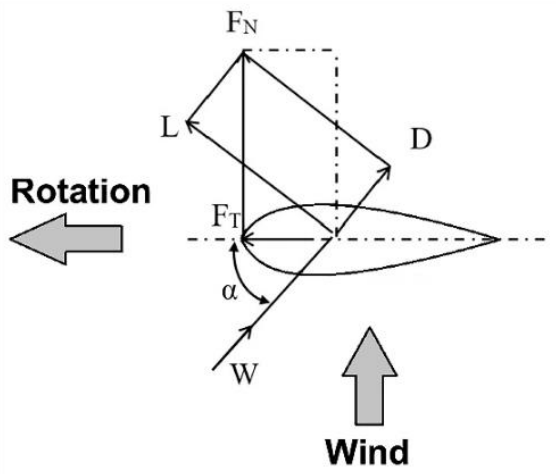

Fig. 2. The aerodynamic force components influencing airfoil section.

In the upstream section, for every blade element at determined azimuthal angle, the instantaneous torque is found by adding the tangential resulting force component around the center of rotor (Kavade and Ghanegaonkar, 2019; Svorcan et al. 2013):

$$
Q(\theta)=\frac{1}{2} C_{T} \rho c R \Delta h W_{u p}^{2}
$$

By summing up the torques of every blade at every azimuthal location, the total mean instantaneous torque of whole upstream section is determined from (Kanyako and Janajreh, 2014):

$$
Q_{u p_{a v}}=\frac{N}{2 \pi} \int_{-\pi / 2}^{\pi / 2} Q(\theta) d \theta
$$

The mean torque coefficient $C_{q u p}$ of upstream half is presented as (Hashem and Mohamed, 2018):

$$
C_{q u p_{a v}}=\frac{Q_{u p_{a v}}}{\frac{1}{2} \rho A R V_{\infty}^{2}}
$$

where $H$ represents the blade height, and $A=2 R H$ indicates the rotor swept area. Accordingly, the upstream power coefficient can be defined with regard to mean torque coefficient $\left(C_{q u p_{a v}}\right)$ and turbine tip speed ratio $\left(\lambda=\omega R / V_{\infty}\right)$ by (Kavade and Ghanegaonkar, 2019):

$$
C_{P u p}=\lambda \cdot C_{q u p} a v
$$

Similar equations are obtained for the downstream section and the parameters are indexed by a prime symbol " , " or with (dw). One can consider the weaknesses of the DMST model as the drag and lift coefficients demands as a function of Reynolds number and the angle of attack. The airfoil reliable data affects the procedure accuracy, particularly in lower Reynolds numbers and higher attack angles. It is, therefore, recommended to use experimental data to decrease calculation errors. In our research, therefore, the experimentally obtained results for symmetrical NACA airfoils of Sheldahl and Klimas
(1981) were utilized at various Reynolds numbers in the whole range of angles of attack $\left(0-180^{\circ}\right)$.

\subsection{DMST Model Developed for Performance Prediction of Darrieus VAWTs}

Figure 3 shows that adjacent and parallel stream tubes are partitioned into vertical and horizontal section in Darrieus VAWT space in the DMST model and the upstream and downstream induced velocities varied for every stream tube section. The flowchart depicted in Fig. 4 represents the overall process of calculations of the DMST model for the Darrieus straight-bladed VAWTs. The first step of the introduced procedure is to enter the primary parameters and describe the space division of VAWT. For the Darrieus VAWT, the geometrical parameters of design such as rotor height $H$, rotor radius $R$, blade chord $c$, lift and drag coefficients airfoil data, and the blades number $N$ are entered. Additionally, the operational parameters (for example, tip speed ratio $\lambda$, free wind velocity $V_{\infty}$, kinematic viscosity of air $v$ and air density $\rho$ ) are introduced to the model. By divided $\left(180^{\circ}\right)$ on the blade azimuth angle variation $\Delta \theta$, the horizontal VAWT space direction is partitioned and the overall number of stream tubes in each horizontal set equals $\left(N_{h}=180^{\circ} / \Delta \theta\right)$. In the vertical space direction of Darrieus straight-bladed VAWTs, every stream tube in one set of vertical stream tubes has the similar behavior as other stream tubes in this set due to the straight blades. Consequently, every blade element $\Delta h$ has the similar instantaneous torque and forces as other elements belonging to the same vertical set. Accordingly, the turbine length $H$ rather than that of stream tube height $\Delta h$ can be considered to calculate the set of vertical stream tubes at a defined azimuthal location $\theta$. As displayed in the flowchart of Fig.4, the next step in this process is to calculate the power coefficient of upstream half $C_{P}$ up and downstream half $C_{P_{d w}}$ of the Darrieus rotor for every tip speed ratios $\lambda$, corresponding to every rotational speed $\left(\omega=\lambda V_{\infty} / R\right)$ with constant values for rotor radius $R$ and free wind velocity $V_{\infty}$. According to Fig. 4, calculating the upstream section is equal to coming upon upstream induction factor $a u$ via blade element momentum theory for all upstream tubes in this section for determining the total momentum transfer between the blades and the wind. Since it is an iterative procedure, the initial induction factor is guessed by the script. Then, it changes the induction factor until the momentum lost by the wind is equal to the momentum acquired by the blades at the same position. Initially, every upstream tube receives an $a u$ value of one. This is followed by calculation of the local relative velocity $W_{u p}$, the upstream induced velocity $V_{a u}$, the local blade Reynolds number $R e_{u p}$, and the local attack angle $\alpha_{u p}$. Next, the lift $C_{L}$ and drag $C_{D}$ coefficients are obtained by consideration of the local blade Reynolds number $R e_{u p}$ and the local attack angle $\alpha_{u p}$ among the experimentally measured data for the chosen airfoil type through a 2D interpolation. Thereafter, one can calculate the tangential and normal coefficients $\left(C_{T}\right.$ and $C_{N}$, respectively). Thus, to determine the new upstream 

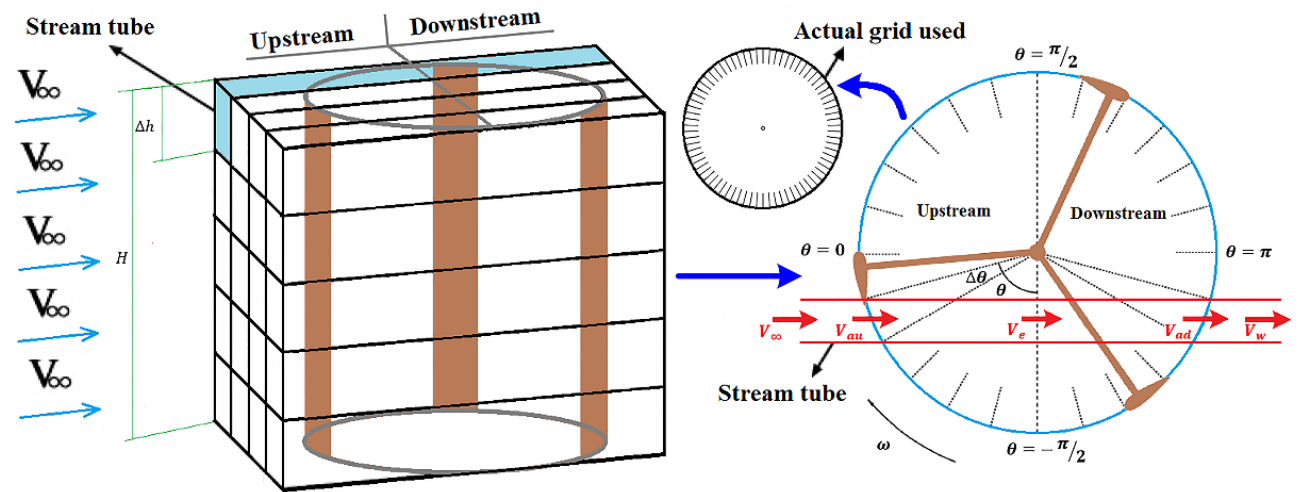

Fig. 3. Sketch of space division for DMST model developed in Darrieus VAWTs.

induction factor $a u_{n e w}, F_{u p}$ that reflects the upwind conditions is computed. After comparing au and $a u_{n e w}$, the difference between these two upstream induction factors is considered an error. In case the error is beyond the specified error tolerance, i.e. $10^{-4}$, a new induction factor $a u_{n e w}$ is considered as a primary value for $a u$ and the entire procedure restarts for this upstream tube, until reaching an error within the error tolerance range. Thereafter, to determine the instantaneous torque at the determined azimuthal location $Q(\theta)$, the tangential and normal aerodynamic forces, $F_{T}(\theta)$ and $F_{N}(\theta)$, of the same upstream tube are estimated. The average torque coefficient $C_{q u p_{a v}}$ and the overall average instantaneous torque $Q_{u p_{a v}}$ of upstream half rotor are computed after processing the all upstream tubes. Therefore, at the chosen tip speed ratio $\lambda$, one can obtain the power coefficient of the upstream section $C_{P_{u p}}$. Afterwards, this process is reiterated for the downwind section (Fig. 4). In this section, the obtained value of $a u$ in every upstream tube is used as an initial value of $a d$ in each versus the downstream tube, and the equilibrium velocity, presented in Eq. (2), is applied for the secondary actuator disk as the free velocity in downstream parts of the stream tubes. Likewise, by employing the blade element momentum theory for all downstream tubes, one can determine the downstream induction factor, $a d$, also through an iterative process with a residue of $10^{-4}$ order. Eventually, after processing the whole downstream tubes, the power coefficient is computed for the downstream section $C_{P d w}$. The final step would be to calculate the total of power coefficient $C_{P}$ of Darrieus rotor at the chosen tip speed ratio via summation of the power coefficient of the downstream section $C_{P_{d w}}$ and upstream section $C_{P \text { up }}$. In order to complete the performance curve of the Darrieus VAWT, the entire steps (Fig. 4) are reiterated for all tip speed ratios $\lambda$.

\subsection{Validation of DMST Model Developed for Performance Prediction of Darrieus VAWTs}

For validating the code developed in MATLAB, a comparison was made between the numerical results of the current model and those of aerodynamic computational model for H-Darrieus wind turbine, named CARDAAV Code and utilized by Paraschivoiu et al. (2009). The same approach was taken by the CARDAAV model on the basis of DMST model by considering a section of the rotor in stream tubes and assumes each of both blade elements specified by a certain stream tube as an actuator disk. Disk 1 characterizes the upwind blade component whereas disk 2 is representative of the downwind blade component. The momentum conservation constitutes the basis of the actuator disk theory; thus, in order to calculate the force imposed on the disks, the wind velocities need to be known. Table 1 lists the specifications of the turbine utilized in this reference. It is worth noting that due to unavailability of the numerical results from the CARDAAV model, the reference data were obtained by reading a plot at tip speed ratios of 1.510 with step value of 0.5 . Thus, the code developed was run for the same values of tip speed ratio obtained from a plot to acquire data points from the DMST model designed in this research and to make a comparison between them and the reference data. Fig. 5 depicts the comparison of power coefficient results. At the selected tip speed ratios between reference values and computed power coefficients, the maximal relative error is $2.6 \%$, indicating a proper concordance between the reference and computed values.

Table 1 VAWT specifications adopted in Paraschivoiu et al. (2009)

\begin{tabular}{|l|c|}
\hline \multicolumn{2}{|c|}{ Paraschivoiu et al. } \\
\hline Blades number, $N(-)$ & 0.2 \\
\hline Turbine height, $H(m)$ & 6 \\
\hline Rotational speed, $\omega(\mathrm{rpm})$ & 125 \\
\hline Turbine radius, $R(\mathrm{~m})$ & 3 \\
\hline Airfoil section type & NACA 0015 \\
\hline
\end{tabular}




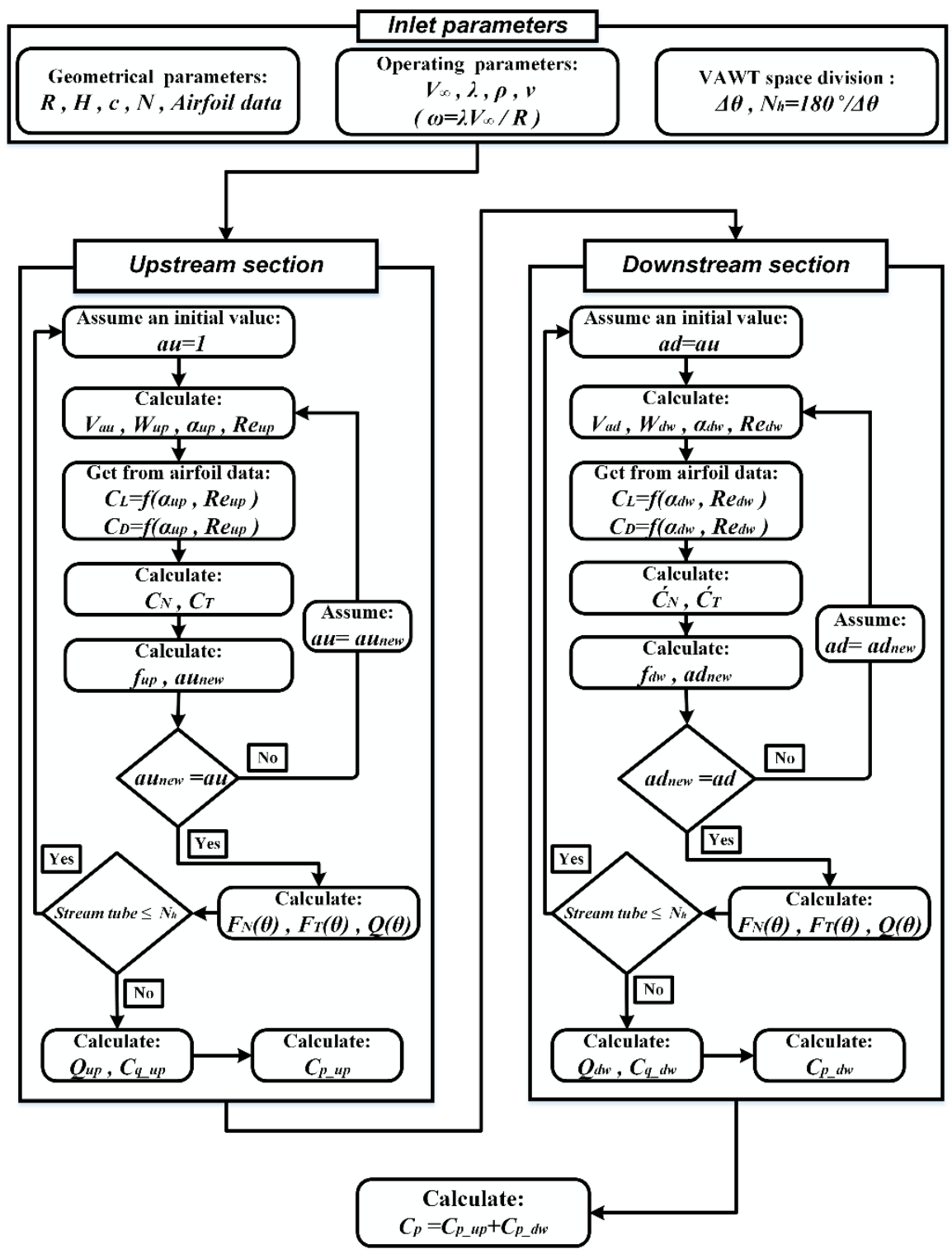

Fig. 4. Flowchart of DMST model developed for performance prediction of Darrieus VAWTs.

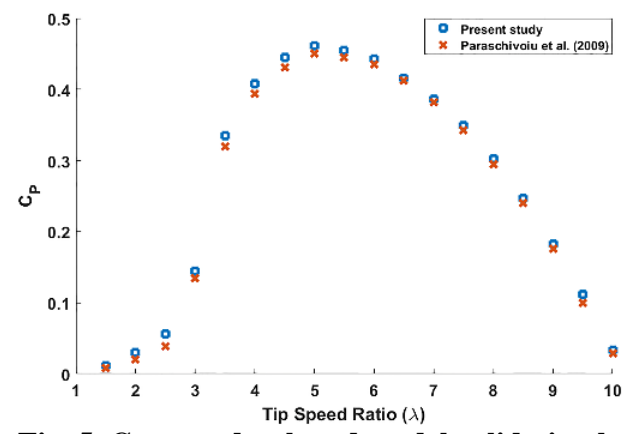

Fig. 5. Current developed model validation by results obtained from utilized model by Paraschivoiu et al. (2009).

\subsection{DMST Model Developed for Performance Prediction of Gorlov VAWTs}

The blades are curved for Gorlov-type VAWTs, which is contrary with the design of Darrieus straight-bladed VAWTs. Thus, while the Gorlov rotor rotating, every blade passes in some azimuth angles, as it is located partly in the upwind and partly in the downwind in accordance with the blades helical angle (Fig. 6). For the same reason, the prior DMST model employed in Darius rotors is not applicable for prediction of the Gorlov rotors aerodynamic performance. Therefore, the DMST model is designed such that it fits the helical blades. The flowchart of DMST aerodynamic prediction model designed for Gorlov VAWTs is illustrated in Fig. 7. The first step begins with inserting the parameters and definition of the Gorlov VAWT space division. According to previous description in the Darrieus-type, the inserted inputs include the same operating and geometric designing parameters beside the helical angle parameter $\Psi$ characterizing the Gorlov rotor. In the Gorlov VAWT space division, the number of horizontal division in every 


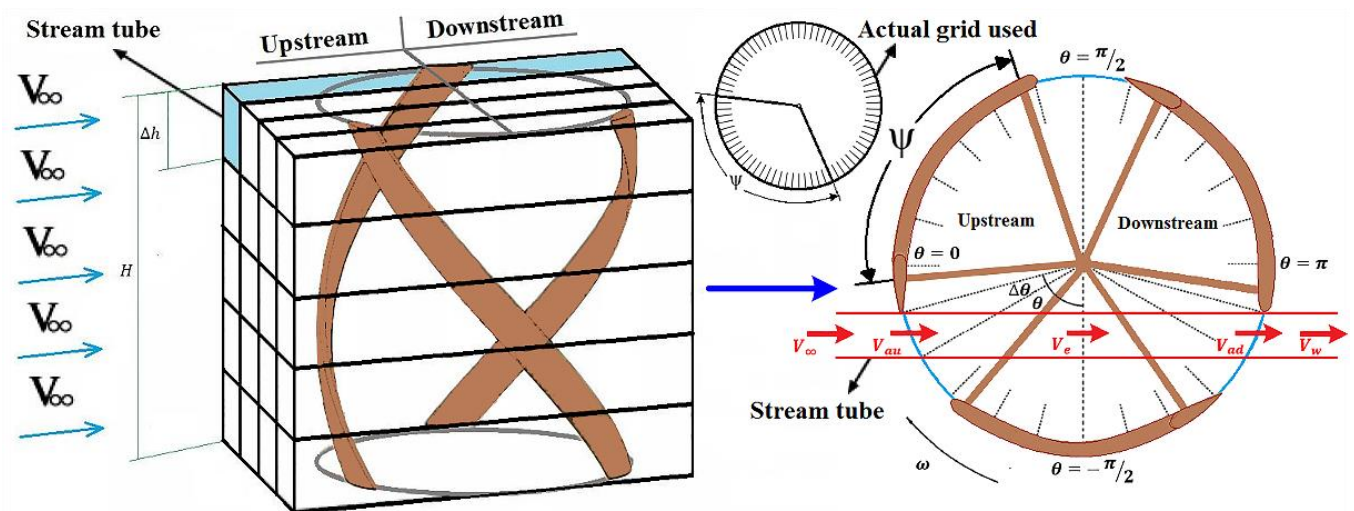

Fig. 6. Sketch of space division for DMST model developed in Gorlov VAWTs.

horizontal set equals $N_{h}=180^{\circ} / \Delta \theta$, while that of vertical divisions in every vertical set equals $N_{v}=$ $\Psi / \Delta \theta$ that for Gorlov VAWTs, it is dependent on the blade azimuth angle variations and the helical angles. Accordingly, at the designated helical angle $\Psi$, the height of every stream tube would be equal to $\Delta h=H / N_{v}$ for covering the behavior of curved blade in every azimuthal angle position. As depicted in the flowchart of Fig. 7, the second step includes calculation of the normal aerodynamic force $F_{N}(\theta)$, the tangential aerodynamic force $F_{T}(\theta)$ and the instantaneous torque $Q(\theta)$ in all the horizontal upstream and downstream tubes at azimuthal locations with the length of blade element $\Delta h$ for each horizontal set at each tip speed ratio $\lambda$. To obtain $a u$ and $a d$ in the upstream and downstream tubes, the iterative approach with an error tolerance of $10^{-4}$ by considering an initial au value of one in every upstream tube and assuming the initial value of $a d$ in every downstream tube equivalent to calculated $a u$ in its opposite upstream tube. When the aerodynamic behavior is calculated for all the stream tubes in horizontal set, the values of components of aerodynamic forces and instantaneous torques are saved, and then all the computation starts again for next set of the horizontal stream tubes. In the third step, therefore, the instantaneous torque and forces generated at a stipulated azimuthal location $\theta$ by a single helical blade are calculable by summation of the instantaneous torque and forces of every vertical stream tube covering the behavior of curved blade using the equations given below:

$$
\begin{aligned}
& F_{N}(\theta)=\sum_{i=1}^{2 N_{h}} \sum_{i}^{i+N_{v}} F_{N_{i}}(\theta) \\
& F_{T}(\theta)=\sum_{i=1}^{2 N_{h}} \sum_{i}^{i+N_{v}} F_{T_{i}}(\theta) \\
& Q(\theta)=\sum_{i=1}^{2 N_{h}} \sum_{i}^{i+N_{v}} Q_{i}(\theta)
\end{aligned}
$$

Thereafter, in the subsequent step, as shown in the flowchart of Fig. 7, the average torque coefficient and the overall average instantaneous torque of the Gorlov VAWT can be expressed as:

$$
\begin{aligned}
Q_{a v} & =\frac{N}{2 \pi} \int_{-\pi / 2}^{3 \pi / 2} Q(\theta) d \theta \\
C_{q_{a v}} & =\frac{Q_{a v}}{\frac{1}{2} \rho A R V_{\infty}{ }^{2}}
\end{aligned}
$$

Therefore, in the final step, the total Gorlov rotor power coefficient shall be:

$$
C_{P}=\lambda \cdot C_{q_{a v}}
$$

To predict the aerodynamic performance curve at various tip speed ratios of Gorlov VAWT, the entire steps represented in Fig. 7 are reiterated for every tip speed ratio $\lambda$.

\subsection{Validation of DMST Model Developed for Performance Prediction of Gorlov VAWTs}

The DMST model designed for Gorlov VAWTs was validated through comparison of the results obtained with the experimental results derived from the wind tunnel test QR5 (Quiet Revolution 5) for the configuration of twisted helical blades mentioned by Scheurich et al. (2010). In this paper, experimental data is used in validation process of the DMST model designed for Gorlov VAWTs due to unavailability of a computational model on the basis of DMST for Gorlov VAWTs in the literature. Hence, this study is the first one to develop the model of DMST aerodynamic performance prediction for Gorlov VAWTs. Although the results of the designed DMST model and those of experiments show a good agreement in lower tip speed ratios (Fig. 8), as a result of the wake expansion effects, some differences are found between the two results in terms of high tip speed ratios. In the introduced model, the velocity is reduced only by the energy extracted via the actuator disk from the wind. In practice, the stream tubes upstream of the rotor and downstream in the wake regime need enlargements, which may lead to consequent reduction of velocity. Thus, given the momentum theory, it is observed that the expansion of the stream tube becomes more noticeable at higher tip speed ratios, as this is where the maximum reduction rate occurs for the velocities induced from expansion effects of the stream tube. 


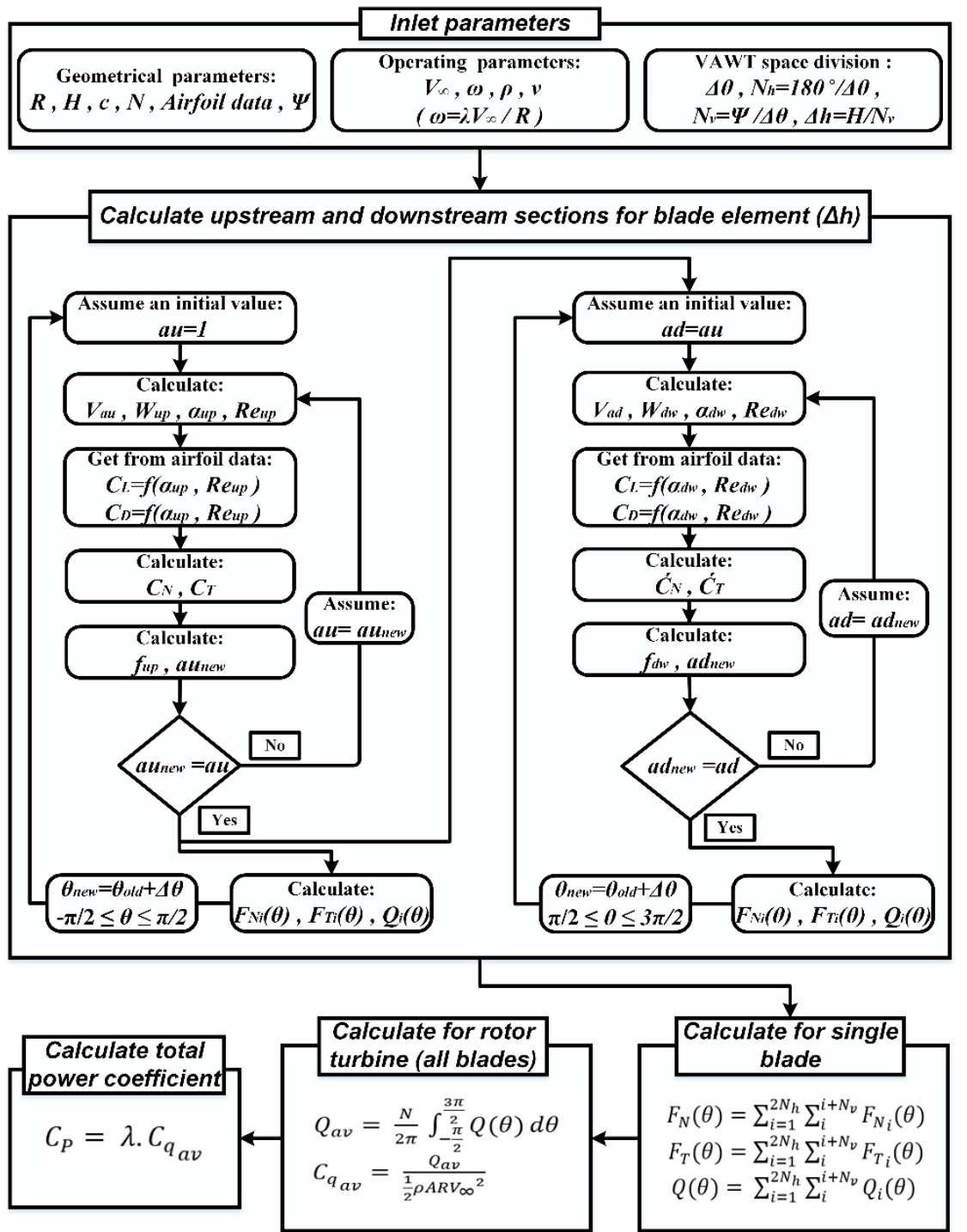

Fig. 7. Flowchart of DMST model developed for performance prediction of Gorlov VAWTs.

Nonetheless, a maximal relative error of $10.1 \%$ between the computed power coefficients and values of experiments at different values of tip speed ratio exists. Hence, the capability of the current code in presenting good predictions of aerodynamic performance for Gorlov VAWTs is confirmed.

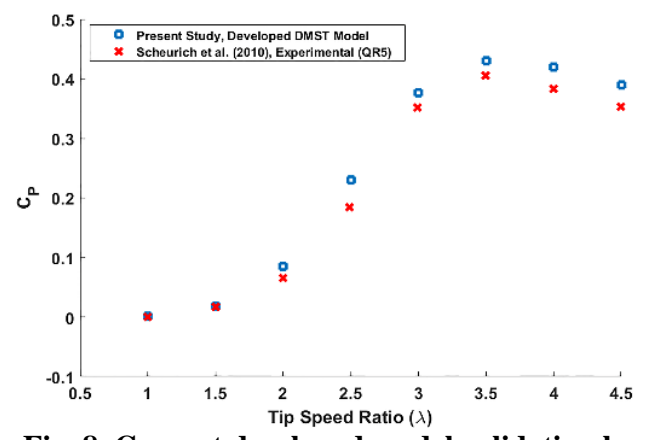

Fig. 8. Current developed model validation by experimental results of QR5 wind tunnel.

\section{RESULTS AND DISCUSSION}

The results obtained from the designed DMST models for Gorlov and Darrieus VAWTs are discussed in this section. Table 2 lists VAWTs characteristics for the present study.

Comparison was made between Gorlov and Darrieus straight-bladed VAWTs with regard to aerodynamic performance and torque coefficients curves.

\subsection{Power Coefficient $\left(C_{P}\right)$}

Figure 9 displays power coefficients calculated for both VAWT types at various tip speed ratios. Figure 9 obviously shows that $C_{P}$ values generation by Gorlov VAWT are less, particularly at higher $\lambda$; but, the rate of reduction is quite slow. Additionally, it is also obvious that the optimum $\lambda=3.5$, where the peak $C_{P}$ takes place for both Gorlov and Darrieus VAWTs, is the same. Besides, a negative value can be seen for the value of the power coefficient at the 
tip speed of 6.5 and higher. This observation can be described from Fig. 10 indicating the attack angle variations as a function of azimuthal angle at different tip speed ratios. It is evident that the value of the angle of attack rises with increasing the azimuth angle until reaching its maximal value, after which it drops with rising the azimuth angle. For a fixed value of azimuth angle, however, the angle of attack decreases with increasing the tip speed ratio until becoming quite small at high tip speed ratios (Fig. 10). Additionally, as the rotational speed of the turbine is increased, the flow encounters greater effective blockade. Moreover, the viscous effects and friction are predominant at greater tip speed ratios resulting in reduced total turbine performance. At higher tip speed ratios, the entire above reasons lead to negative power coefficient.

Table 2 VAWTs characteristics for the present study

\begin{tabular}{|c|c|c|}
\hline $\begin{array}{c}\text { Turbine } \\
\text { characteristics }\end{array}$ & $\begin{array}{c}\text { Darrieus } \\
\text { VAWT }\end{array}$ & $\begin{array}{c}\text { Gorlov } \\
\text { VAWT }\end{array}$ \\
\hline Chord length, $c(m)$ & 0.2 & 0.2 \\
\hline $\begin{array}{c}\text { Blades number, } N \\
(-)\end{array}$ & 3 & 3 \\
\hline $\begin{array}{c}\text { Turbine height, } H \\
(m)\end{array}$ & 3 & 10 \\
\hline $\begin{array}{c}\text { Free wind velocity, } \\
V_{\infty}(m / s)\end{array}$ & 10 & 120 \\
\hline Helical angle, $\psi\left({ }^{\circ}\right)$ & $\begin{array}{c}\text { Straight } \\
\text { blades }\end{array}$ & 1.5 \\
\hline $\begin{array}{c}\text { Turbine radius, } R \\
(m)\end{array}$ & 1.5 & NACA 0018 \\
\hline Airfoil section type & NACA 0018 \\
\hline
\end{tabular}

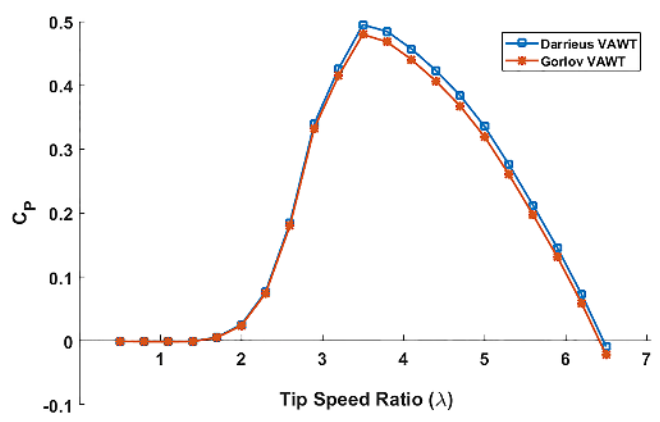

Fig. 9. Comparing aerodynamic performance $\left(C_{P}\right)$ of Gorlov and Darrieus VAWTs.

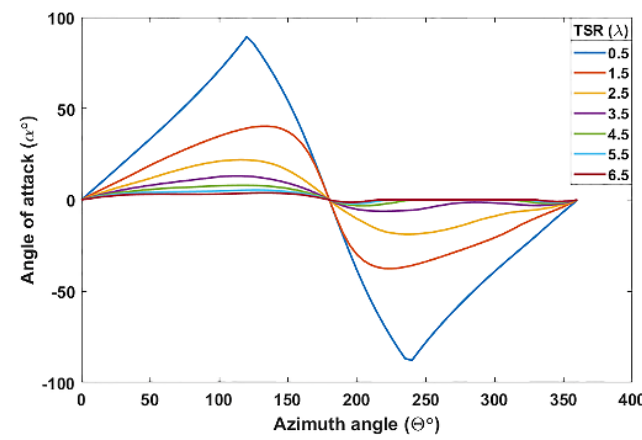

Fig. 10. Variation of attack angle at various tip speed ratios as a function of azimuthal angle.

\subsection{Power Fluctuation $\left(C_{q}\right)$}

Figure 11 compares the fluctuations of torque coefficient $C_{q}$ during a rotor rotation at the optimum tip speed ratio value $\lambda=3.5$ where the maximum $C_{P}$ happens for both Gorlov and Darrieus rotors. The $C_{q}$ curves of the straight blades in Darrieus VAWT and the helical blades in Gorlov VAWT are shown in Fig. 11(a) and 11(b), respectively. One can observe lower fluctuations with more efficient $C_{q}$ curve in one cycle for every helical blade. Also, $C_{q}$ curve with lower decreases to zero values is observable for Gorlov blade compared to Darrieus blade. This can be attributable to that a section from the helical blade always exists in optimum location in terms of the relative wind. In addition, the comparison presented in Fig. 11 between $C_{q}$ for Gorlov and Darrieus rotors (all blades) represents smoother and more uniformly distributed $C_{q}$ curve for Gorlov rotor than Darrieus rotor across all rotation ranges, resulting in better aerodynamic performance and lower fluctuations in torque coefficient curve.

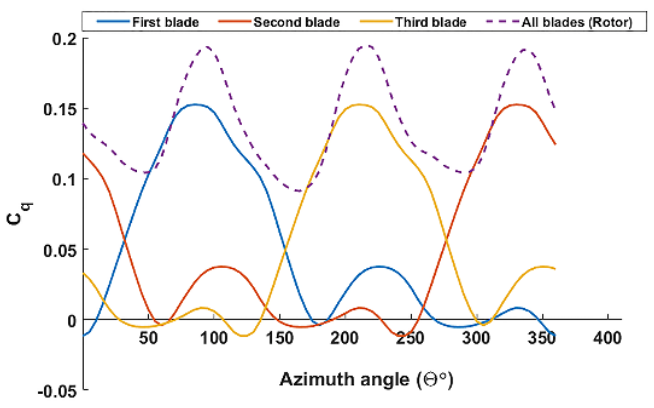

(a) Darrieus VAWT rotor

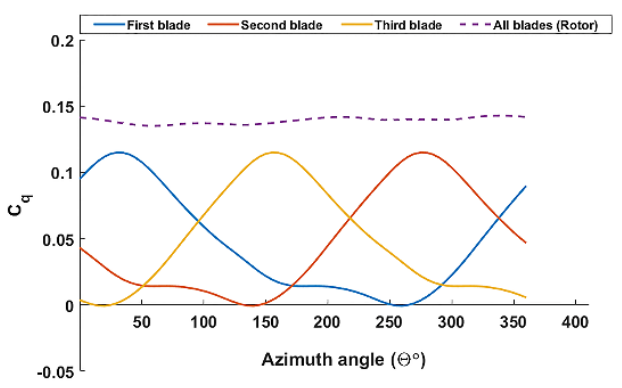

(b) Gorlov VAWT rotor

Fig. 11. Comparing power fluctuation $\left(C_{q}\right)$ of Gorlov VAWT and Darrieus VAWT rotors at $\lambda=3.5$.

\section{Conclusion}

The present research develops the fast aerodynamic calculations DMST models for both Gorlov and Darrieus straight-bladed VAWT types. Subsequently, both VAWTs were evaluated from points of the aerodynamic performances $\left(C_{P}\right)$ and the power fluctuation $\left(C_{q}\right)$, followed by results comparison. According to the comparison results, the peak of $C_{P}$ for Gorlov-type decreased slightly in comparison to $C_{P}$ peak for Darrieus-type. 
Nevertheless, in one cycle, the $C_{q}$ curve for every Gorlov blade was lower decline to zero values than the $C_{q}$ curve for every Darrieus blade. This leads to smoother and lower variations $C_{q}$ curve for Gorlov rotor than Darrieus rotor. It can, therefore, be concluded that the Gorlov VAWT has a better aerodynamic performance.

\section{REFERENCES}

Abdul Akbar, M. and V. Mustafa (2016). A new approach for optimization of Vertical Axis Wind Turbines. Journal of Wind Engineering and Industrial Aerodynamics 153, 34-45.

Abu-Hamdeh, N. H. and K. H. Almitani (2017). Construction and numerical analysis of a collapsible vertical axis wind turbine. Energy Conversion and Management 151, 400-413.

Alaimo, A., A. Esposito, A. Messineo, C. Orlando, D. Tumino, A. Alaimo, A. Esposito, A. Messineo, C. Orlando and D. Tumino (2015). 3D CFD Analysis of a Vertical Axis Wind Turbine. Energies 8(4), 3013-3033.

Amiri, M., M. Kahrom and A. R. Teymourtash (2019). Aerodynamic analysis of a threebladed pivoted savonius wind turbine: Wind tunnel testing and numerical simulation. Journal of Applied Fluid Mechanics 12(3), 819-829.

Balduzzi, F., A. Bianchini, G. Ferrara and L. Ferrari (2016). Dimensionless numbers for the assessment of mesh and timestep requirements in CFD simulations of Darrieus wind turbines. Energy 97, 246-261.

Balduzzi, F., J. Drofelnik, A. Bianchini, G. Ferrara, L. Ferrari and M. S. Campobasso (2017). Darrieus wind turbine blade unsteady aerodynamics: a three-dimensional NavierStokes CFD assessment. Energy 128, 550563.

Batista, N. C., R. Melicio and V. M. F. Mendes (2018). Darrieus vertical axis wind turbines: methodology to study the self-start capabilities considering symmetric and asymmetric airfoils. Research on Engineering Structures and Materials 4(3), 189-217.

Battisti, L., G. Persico, V. Dossena, B. Paradiso, M. Raciti Castelli, A. Brighenti and E. Benini (2018). Experimental benchmark data for $\mathrm{H}$ shaped and troposkien VAWT architectures. Renewable Energy 125, 425-444.

Bedon, G., M. Raciti Castelli and E. Benini (2014). Proposal for an innovative chord distribution in the Troposkien vertical axis wind turbine concept. Energy 66, 689-698.

Cheng, Q., X. Liu, H. S. Ji, K. C. Kim, B. Yang, Q. Cheng, X. Liu, H. S. Ji, K. C. Kim and B. Yang (2017). Aerodynamic Analysis of a Helical Vertical Axis Wind Turbine. Energies
10(4), 575.

Derakhshan, S., M. Moghimi and H. Motawej (2018). Development of a mathematical model to design an offshore wind and wave hybrid energy system. Energy Equipment and Systems 6(2), 181-200.

Dilimulati, A., T. Stathopoulos and M. Paraschivoiu (2018). Wind turbine designs for urban applications: A case study of shrouded diffuser casing for turbines. Journal of Wind Engineering and Industrial Aerodynamics 175, 179-192.

Gorlov, A. M. (1995). Unidirectional helical reaction turbine operable under reversible fluid flow for power systems. U.S. Patent 5451137.

Hashem, I. and M. H. Mohamed (2018). Aerodynamic performance enhancements of H-rotor Darrieus wind turbine. Energy 142, 531-545.

Jafari, M., A. Razavi and M. Mirhosseini (2018). Effect of airfoil profile on aerodynamic performance and economic assessment of $\mathrm{H}$ rotor vertical axis wind turbines. Energy 165 , 792-810.

Kanyako, F. and I. Janajreh (2014). Vertical Axis Wind Turbine performance prediction for low wind speed environment. Digest of Technical Papers - InnoTek 2014: IEEE Innovations in Technology Conference 1-10.

Kavade, R. K. and P. M. Ghanegaonkar (2018). Effect of best position blade pitching on power coefficient of VAWT at different tip speed ratio by SST \&amp; DMST model. FME Transactions 46(4), 560-566.

Kavade, R. K. and P. M. Ghanegaonkar (2019). Performance Evaluation of Small-Scale Vertical Axis Wind Turbine by Optimized Best Position Blade Pitching at Different Tip Speed Ratios. Journal of The Institution of Engineers (India): Series C 100(6), 10051014.

Meana-Fernández, A., I. Solís-Gallego, J. M. Fernández Oro, K. M. Argüelles Díaz and S. Velarde-Suárez (2018). Parametrical evaluation of the aerodynamic performance of vertical axis wind turbines for the proposal of optimized designs. Energy 147, 504-517.

Moghimi, M. and H. Motawej (2020). Developed DMST model for performance analysis and parametric evaluation of Gorlov vertical axis wind turbines. Sustainable Energy Technologies and Assessments 37, 100616.

Moghimi, M., S. Derakhshan and H. Motawej (2018). A Mathematical Model Development for Assessing the Engineering and Economic Improvement of Wave and Wind Hybrid Energy System. Iranian Journal of Science and Technology, Transactions of Mechanical Engineering 2018, 1-15. 
Noura, B., I. Dobrev, R. Kerfah, F. Massouh and S. Khelladi (2016). Investigation of the rotor wake of horizontal axis wind turbine under yawed condition. Journal of Applied Fluid Mechanics 9(6), 2695-2705.

Paraschivoiu, I. (1981). Double-multiple streamtube model for Darrieus in turbines. In NASA. Lewis Research Center Wind Turbine Dyn. 1981,19-25.

Paraschivoiu, I. (2002). Wind Turbine Design with Empasis on Darrieus Concept. Polytechnic International Press.

Paraschivoiu, I., O. Trifu and F. Saeed (2009). HDarrieus wind turbine with blade pitch control. International Journal of Rotating Machinery 2009, 505343.

Peng, Y.-X., Y.-L. Xu, S. Zhan and K.-M. Shum (2019). High-solidity straight-bladed vertical axis wind turbine: Aerodynamic force measurements. Journal of Wind Engineering and Industrial Aerodynamics 184, 34-48.

Rogowski, K., M. O. L. Hansen and P. Lichota (2018). 2-D CFD computations of the twobladed Darrieus-type wind turbine. Journal of Applied Fluid Mechanics 11(4), 835-845.

Saeidi, D., A. Sedaghat, P. Alamdari and A. A. Alemrajabi (2013). Aerodynamic design and economical evaluation of site specific small vertical axis wind turbines. Applied Energy 101, 765-775.

Sagharichi, A., M. Zamani and A. Ghasemi (2018). Effect of solidity on the performance of variable-pitch vertical axis wind turbine. Energy 161, 753-775.

Scheurich, F., T. M. Fletcher and R. E. Brown (2010). The influence of blade curvature and helical blade twist on the performance of a vertical-axis wind turbine. In: 29th ASME Wind Energy Symposium 2010, 1-16.

Sheldahl, R. E. and P. C. Klimas (1981). Aerodynamic Characteristics of Seven Symmetrical Airfoil Sections through 180Degree Angle of Attack for Use in Aerodynamic Analysis of Vertical Axis Wind Turbines. Albuquerque, NM, and Livermore, CA (United States), No. SAND-80-2114.
Strickland, J. (1975). The Darrieus Turbine, A Performance Prediction Method Using Multiple Stream Tubes. Sandia Laboratories, SAND (No. SAND-75-0431).

Svorcan, J., S. Stupar, D. Komarov, O. Peković and I. Kostić (2013). Aerodynamic design and analysis of a small-scale vertical axis wind turbine. Journal of Mechanical Science and Technology 27(8), 2367-2373.

Tahani, M., N. Babayan, S. Mehrnia and M. Shadmehri (2016). A novel heuristic method for optimization of straight blade vertical axis wind turbine. Energy Conversion and Management 127, 461-476.

Templin, R. J. (1974). Aerodynamic performance theory for the NRC vertical-axis wind turbine. NASA STI/RECON Technical Report $N$ 76(Jun), LTR-LA-160.

UN News Centre, 2015. UN adopts new Global Goals, charting sustainable development for people and planet by 2030. United Nations Dep. Econ. Soc. Aff. In: http://www.un.org/en/development/desa/new s/sustainable/un-adopts-new-global-goals. html\#more-15178. (Accessed 18 Apr 2019)

Zamani, M., M. J. Maghrebi and S. R. Varedi (2016). Starting torque improvement using J-shaped straight-bladed Darrieus vertical axis wind turbine by means of numerical simulation. Renewable Energy 95, 109-126.

Zhao, Z., S. Qian, W. Shen, T. Wang, B. Xu, Y. Zheng and R. Wang (2017). Study on variable pitch strategy in H-type wind turbine considering effect of small angle of attack. Journal of Renewable and Sustainable Energy 9(5), 053302.

Zhu, H., W. Hao, C. Li and Q. Ding (2019). Numerical study of effect of solidity on vertical axis wind turbine with Gurney flap. Journal of Wind Engineering and Industrial Aerodynamics 186, 17-31.

Zhu, J. Y. and P. Q. Liu (2018). Effect of pitch angle and reynolds number on aerodynamic characteristics of a small horizontal axis wind rotor. Journal of Applied Fluid Mechanics 11(3), 613-620. 